\title{
Asociación entre comorbilidades cardiometabólicas y enfermedad de Parkinson en población mexicana
}

\author{
Amin Cervantes-Arriaga ${ }^{1,2}$, Óscar Esquivel-Zapata², Emmanuel Escobar-Valdivia1, David García-Romero², \\ Ángel Alcocer-Salas ${ }^{2}$ y Mayela Rodríguez-Violante, \\ ${ }^{1}$ Clínica de Trastornos del Movimiento; ${ }^{2}$ Laboratorio Clínico de Enfermedades Neurodegenerativas. Instituto Nacional de Neurología y Neurocirugía, \\ Ciudad de México, México
}

\section{Resumen}

Antecedentes: La prevalencia de la enfermedad de Parkinson (EP) aumenta a medida que la población envejece. Los estudios han demostrado que algunas comorbilidades cardiometabólicas pudieran estar asociadas con el riesgo o la protección de desarrollar la EP. Se realizó un estudio retrospectivo de casos y controles para analizar la relación entre la EP y las comorbilidades cardiometabólicas. Material y métodos: Se reclutaron sujetos con EP y controles sin EP de forma consecutiva. Se recolectaron datos sobre diabetes mellitus tipo 2, hipertensión arterial sistémica (HTA), dislipidemia e índice de masa corporal. Se llevó a cabo análisis de regresión logística. Resultados: Se incluyeron un total de 781 personas con EP (56,5\% hombres) y 1,000 controles (44,4\% hombres). Después de ajustar por edad y sexo, la HTA se encontró como factor de riesgo independiente (OR 1.32, IC 95\% 1.05-1.67, $p=0.02$ ) y la obesidad como factor protector (OR 0.72, IC 95\% 0.56-0.93, $p=0.01)$. Conclusiones: Los sujetos con HTA tienen un mayor riesgo de tener EP; mientras que los sujetos obesos tienen un menor riesgo de tener EP. La relación entre la enfermedad cardiometabólica, su tratamiento y etiopatogenia de la EP parece ser extremadamente compleja dada la cantidad de datos contradictorios.

PALABRAS CLAVE: Índice de masa corporal. Diabetes mellitus. Dislipidemia. Hipertensión. Enfermedad de Parkinson. Riesgo

\section{Association between cardiometabolic comorbidities and Parkinson's disease in a Mexican population}

\begin{abstract}
Background: The prevalence of Parkinson's disease (PD) increases as the population ages. Studies have shown that some cardiometabolic comorbidities could be associated with risk or protection against developing PD. A retrospective case-control study was carried out to analyze the relationship between $P D$ and cardiometabolic comorbidities. Material and methods: Subjects with $P D$ and controls without $P D$ were consecutively recruited. Data on type 2 diabetes mellitus, systemic arterial hypertension (SAH), dyslipidemia and body mass index were collected. Logistic regression analyses were carried out. Results: A total of 781 subjects with PD (56.5\% male) and 1,000 controls (44.4\% males) were included. After adjusting for age and gender, SAH was found as an independent risk factor (OR 1.32, Cl 95\% 1.05-1.67, $p=0.02$ ), and obesity a protective factor (OR 0.72, CI 95\% 0.56-0.93, $p=0.01$ ). Conclusion: Subjects with SAH had an higher risk of having PD. while obese subjects had a lower risk of having PD. The relationship between cardiometabolic disease, its treatment, and PD etiopathogenesis appears to be extremely complex given the amount of contradictory data.
\end{abstract}

KEY WORDS: Body mass index. Diabetes mellitus. Dyslipidemia. Hypertension. Parkinson's disease. Risk.

Correspondencia:

*Mayela Rodríguez-Violante

E-mail: mrodriguez@innn.edu.mx

0016-3813/@ 2021 Academia Nacional de Medicina de México, A.C. Publicado por Permanyer. Este es un artículo open access bajo la licencia

Fecha de recepción: 13-05-2021

Fecha de aceptación: 11-10-2021

A.C. Publicado por Permanyer. Este es un artículo open access bajo la licencia CC BY-NC-ND (http://creativecommons.org/licenses/by-nc-nd/4.0/).
Gac Med Mex. 2021;157:645-650

Disponible en PubMed

Dispo.com (1) 


\section{Introducción}

La prevalencia de la enfermedad de Parkinson (EP) aumenta a medida que la población envejece. En México, la densidad de incidencia notificada entre 2014 y 2017 fue de 9.48 por 100,000 personas-año, lo que resulta en un problema de salud pública'. Los mecanismos fisiopatológicos de la EP no se comprenden completamente. Se ha sugerido que las vías superpuestas, incluidas alteraciones mitocondriales, la neuroinflamación y la agregación de proteínas, interactúan en la patogénesis y el desarrollo de la enfermedad ${ }^{2}$.

La interacción entre diferentes factores ambientales y genéticos podría contribuir de manera importante al desarrollo de la $\mathrm{EP}^{3}$. Existe evidencia actual que sugiere que algunas comorbilidades pudieran estar asociadas con el riesgo o con la protección para el desarrollo de la EP ${ }^{4,5}$. Entre estos, los factores de riesgo cardiometabólico principales han despertado gran interés.

Los factores que favorecen un estado proinflamatorio y el metabolismo alterado de los radicales libres convergen en la disfunción mitocondrial, vinculando la neurodegeneración con la resistencia a la insulina en una vía común ${ }^{6}$.

La diabetes mellitus tipo 2 (DM2) se ha relacionado no solo con el riesgo de desarrollar EP, sino también como un factor modificador en la progresión y la historia natural de la enfermedad ${ }^{7}$, . Además, la mayoría de los estudios epidemiológicos, aunque no todos, han reportado una asociación entre la DM2 y un mayor riesgo de $\mathrm{EP}^{9-11}$.

Los estudios epidemiológicos que abordan la asociación entre la hipertensión arterial (HTA) y la EP han sido contradictorios, con algunos estudios identificando a la HTA como un riesgo y otros como un factor protector $^{12,13}$. De manera similar, la dislipidemia ha sido un tema controvertido en cuanto a si es un factor de riesgo o de protección para la $\mathrm{EP}^{13,14}$. Finalmente, la asociación entre el índice de masa corporal (IMC) y la EP tampoco ha sido concluyente. Algunos estudios sugieren que no existe una relación significativa entre el IMC y el desarrollo de la EP15,16. Por otro lado, otros estudios que incluyen metaanálisis mostraron que tanto el sobrepeso como el bajo IMC podrían ser un factor de riesgo relevante $e^{17-19}$.

Establecer la interacción de patologías es un punto de partida relevante para identificar posibles áreas de intervención. Con el envejecimiento de la población, aumentará la prevalencia de patologías cardiometabólicas y neurodegenerativas.

El objetivo de este estudio de casos y controles es evaluar la relación entre la EP y las principales comorbilidades cardiometabólicas en una muestra de América Latina para enriquecer el conocimiento actual.

\section{Materiales y métodos}

Se incluyeron pacientes consecutivos diagnosticados con EP utilizando los Criterios de diagnóstico clínico para la enfermedad de Parkinson de la Sociedad de Trastornos del Movimiento ${ }^{20}$ en la Clínica de Trastornos del Movimiento del Instituto Nacional de Neurología y Neurocirugía (Ciudad de México, México) entre 2016 y 2019. Se excluyeron los sujetos con hipertensión secundaria, enfermedad pancreática, hipotiroidismo o hipertiroidismo, o cualquier tipo de enfermedad renal.

Los controles, de 50 años de edad o más, se reclutaron en la sala de espera de la consulta externa. Todos los sujetos aceptaron participar y otorgaron su consentimiento firmado. Los mismos criterios de exclusión fueron considerados para el grupo de control. Además, los antecedentes personales o de familiares en primer grado de enfermedad neurológica o neuropsiquiátrica también fueron considerados como criterios de exclusión para este grupo.

La evaluación de la historia cardiometabólica se recogió mediante un cuestionario semiestructurado que constaba de varias partes. En primer lugar, se recopilaron variables sociodemográficas que incluían edad y sexo. A todos los sujetos se les interrogó sobre el diagnóstico conocido de DM2, duración de la enfermedad y cualquier tratamiento hipoglucemiante. La segunda parte evaluó la presencia del diagnóstico de HTA, la duración de la enfermedad y cualquier tratamiento antihipertensivo. En la siguiente parte, se evaluó el diagnóstico de dislipidemia incluyendo la duración de la enfermedad y el uso de fármacos hipolipemiantes. Si los sujetos tomaban un fármaco hipoglucemiante, antihipertensivo o hipolipemiante sin una indicación conocida, se profundizó en la historia clínica para conocer la indicación. Se excluyó a los sujetos que tomaban cualquiera de estos fármacos sin un motivo conocido. En caso necesario, se proporcionó a los sujetos una lista completa de medicamentos para ayudarlos a recordar el nombre.

La última parte evaluó las variables antropométricas, incluida la altura (en metros) y el peso (en 
kilogramos) para el cálculo del IMC. Los sujetos fueron categorizados según su IMC en bajo peso, normal, sobrepeso y obesidad según los estándares internacionales.

El protocolo del estudio fue aprobado por el comité de ética local (121/19) y todos los participantes dieron su consentimiento informado por escrito.

\section{Análisis estadístico}

Se utilizó la prueba de Kolmogorov-Smirnoff para probar la normalidad. Todas las variables de interés tuvieron una distribución no paramétrica. Para la comparación de variables cuantitativas se utilizó la prueba $\mathrm{U}$ de Mann-Whitney. Las variables cualitativas se evaluaron mediante la prueba de chi cuadrada. Las variables con diferencias estadísticamente significativas en el análisis bivariante se incluyeron en el análisis multivariante.

Para los análisis de regresión logística, la presencia de EP se definió como variable dependiente. Se utilizó la regresión logística simple para estimar las razones de momios (OR) de cada variable independiente, seguida de una regresión logística multivariante por pasos. Se utilizó la prueba de Hosmer-Lemeshow para determinar la bondad de ajuste. Se estableció un valor de $p<0.05$ para significancia estadística. Los análisis estadísticos se realizaron con SPSS, versión 17 (SPSS Inc., Chicago, IL, EE.UU.).

\section{Resultados}

Se incluyeron un total de 781 personas con EP ( $56.5 \%$ hombres) y 1,000 controles $(44.4 \%$ hombres). La tabla 1 muestra la comparación completa de las principales variables entre ambos grupos.

La HTA y la dislipidemia fueron más frecuentes en el grupo de EP en comparación con los controles. No se encontró diferencia estadísticamente significativa en la prevalencia de DM2 entre grupos, aunque la DM2 fue algo más frecuente y con tendencia a la significancia estadística en el grupo de EP. Finalmente, los sujetos con EP tenían un IMC más bajo en comparación con los controles. En el grupo de EP, el peso normal fue más frecuente en comparación con los controles. Por otro lado, la obesidad fue más frecuente en los controles que en el grupo de EP.

En el análisis bivariante, mediante regresión logística simple, la HTA (OR: 1.62; intervalo de confianza del 95\% [IC 95\%]: 1.31-2.01; $p<0.001$ ) y la dislipidemia (OR: 1.31; IC 95\%: 1.01-1.70; $p=0.03$ ) se
Tabla 1. Comparación de las principales variables entre controles y los sujetos con enfermedad de Parkinson (EP)

\begin{tabular}{|c|c|c|c|}
\hline & $\begin{array}{l}\text { Controles } \\
(n=1,000)\end{array}$ & $\begin{array}{c}\text { EP } \\
(n=781)\end{array}$ & $p$ \\
\hline $\begin{array}{l}\text { Sexo } \\
\text { Masculino n (\%) } \\
\text { Femenino n (\%) }\end{array}$ & $\begin{array}{l}444(44.4) \\
556(55.6)\end{array}$ & $\begin{array}{l}442(56.6) \\
339(43.4)\end{array}$ & $\begin{array}{l}<0.001^{*} \\
<0.001^{*}\end{array}$ \\
\hline $\begin{array}{l}\text { Edad } \\
\qquad \text { Media } \pm D E\end{array}$ & $57.8 \pm 15.4$ & $62.9 \pm 13.2$ & $<0.001^{\dagger}$ \\
\hline $\begin{array}{l}\text { Diagnósticos } \\
\text { Diabetes mellitus } \\
\text { Hipertensión } \\
\text { Dislipidemia }\end{array}$ & $\begin{array}{l}132(13.2 \%) \\
225(22.5 \%) \\
141(14.1 \%)\end{array}$ & $\begin{array}{c}128(16.4 \%) \\
250(32 \%) \\
139(17.8 \%)\end{array}$ & $\begin{array}{c}0.06^{*} \\
<0.01- \\
0.03-\end{array}$ \\
\hline $\begin{array}{l}\text { IMC } \\
\text { Media } \pm \text { DE } \\
\text { Normal (\%) } \\
\text { Bajo peso (\%) } \\
\text { Sobrepeso (\%) } \\
\text { Obesidad (\%) }\end{array}$ & $\begin{array}{c}27.1 \pm 4.8 \\
325(32.5 \%) \\
16(1.6 \%) \\
401(40.1 \%) \\
258(25.8 \%)\end{array}$ & $\begin{array}{c}26.5 \pm 4.8 \\
292(37.4 \%) \\
20(2.6 \%) \\
302(38.7 \%) \\
167(21.4 \%)\end{array}$ & $\begin{array}{l}<0.01^{\dagger} \\
0.03^{*} \\
0.15^{*} \\
0.54^{*} \\
0.03^{*}\end{array}$ \\
\hline
\end{tabular}

encontraron como factores de riesgo para la EP. Por otro lado, la presencia de obesidad mostró ser un factor protector para la EP (OR: 0.72; IC 95\%: 0.560.93; $p=0.01)$. Además, el sexo femenino también demostró ser un factor protector (OR: 0.61; IC 95\%: $0.51-0.74 ; p<0.001$ ).

Posterior a ajustar por edad y sexo en el análisis multivariante, solo la HTA persistió como factor de riesgo independiente (OR: 1.32; IC 95\%: 1.05-1.67; $\mathrm{p}=0.02$ ) y la obesidad permaneció como factor protector (OR: 0.72; IC 95\%: 0.56-0.93; $p=0.01$ ). En la tabla 2 se muestra una descripción completa del modelo. La presencia de DM2 se incluyó en el modelo debido a fundamentos teóricos, aunque no se alcanzó una significación estadística en el análisis bivariante.

\section{Discusión}

Este es el primer estudio de casos y controles mexicano que evalúa la asociación de las principales comorbilidades cardiometabólicas y la EP. Nuestro estudio no encontró una proporción diferente de DM2 en los pacientes con EP en comparación con los controles, aunque se observó una tendencia. Por otro lado, encontramos que el grupo de EP tenía un perfil diferente, leve pero significativo, en otros factores de riesgo cardiometabólico en comparación con la población general. El grupo de EP tuvo mayor frecuencia 
Tabla 2. Modelo de regresión logística utilizando a la enfermedad de Parkinson como variable dependiente

\begin{tabular}{|c|c|c|c|c|c|c|}
\hline \multicolumn{2}{|c|}{ Modelo } & \multirow{2}{*}{$\begin{array}{c}\text { B } \\
0.024\end{array}$} & \multirow{2}{*}{$\begin{array}{c}\operatorname{Exp}(B) \\
1.024\end{array}$} & \multicolumn{2}{|c|}{ IC 95\% } & \multirow{2}{*}{$\begin{array}{c}p \\
<0.001\end{array}$} \\
\hline 1 & Edad & & & 1.024 & 1.018 & \\
\hline & Sexo femenino & -0.483 & 0.617 & 0.617 & 0.509 & $<0.001$ \\
\hline \multirow[t]{2}{*}{2} & Edad & 0.022 & 1.022 & 1.015 & 1.029 & $<0.001$ \\
\hline & Sexo femenino & -0.487 & 0.615 & 0.507 & 0.745 & $<0.001$ \\
\hline \multirow[t]{5}{*}{3} & HTA & 0.281 & 1.324 & 1.058 & 1.657 & 0.014 \\
\hline & Edad & 0.021 & 1.022 & 1.014 & 1.029 & $<0.001$ \\
\hline & Sexo femenino & -0.486 & 0.615 & 0.508 & 0.745 & $<0.001$ \\
\hline & HTA & 0.272 & 1.313 & 1.047 & 1.645 & 0.018 \\
\hline & DM2 & 0.092 & 1.097 & 0.835 & 1.441 & 0.507 \\
\hline \multirow[t]{5}{*}{4} & Edad & 0.021 & 1.021 & 1.014 & 1.029 & $<0.001$ \\
\hline & Sexo femenino & -0.491 & 0.612 & 0.505 & 0.741 & $<0.001$ \\
\hline & HTA & 0.254 & 1.289 & 1.026 & 1.62 & 0.029 \\
\hline & DM2 & 0.088 & 1.092 & 0.831 & 1.435 & 0.529 \\
\hline & Dislipidemia & 0.143 & 1.154 & 0.884 & 1.506 & 0.293 \\
\hline \multirow[t]{9}{*}{5} & Edad & 0.02 & 1.02 & 1.013 & 1.028 & $<0.001$ \\
\hline & Sexo femenino & -0.491 & 0.612 & 0.505 & 0.742 & $<0.001$ \\
\hline & HTA & 0.281 & 1.324 & 1.052 & 1.666 & 0.017 \\
\hline & DM2 & 0.102 & 1.108 & 0.841 & 1.458 & 0.466 \\
\hline & Dislipidemia & 0.17 & 1.186 & 0.907 & 1.551 & 0.213 \\
\hline & IMC & & & & & 0.033 \\
\hline & Bajo peso & 0.376 & 1.457 & 0.728 & 2.917 & 0.288 \\
\hline & Sobrepeso & -0.184 & 0.832 & 0.665 & 1.04 & 0.106 \\
\hline & Obesidad & -0.329 & 0.72 & 0.555 & 0.933 & 0.013 \\
\hline
\end{tabular}

IC 95\%: intervalo de confianza del 95\%; HTA: hipertensión arterial sistémica: DM2: diabetes mellitus tipo 2; IMC: índice de masa corporal.

de HTA y dislipidemia, y menor frecuencia de obesidad.

Al respecto, nuestro estudio contrasta con algunos datos epidemiológicos publicados basados en estudios prospectivos que han reportado un mayor riesgo de EP en pacientes con DM2. En este sentido, un metaanálisis publicado por Yue, et al. mostró que los estudios prospectivos realizados en diferentes poblaciones pueden variar significativamente ${ }^{9}$. Por ejemplo, un estudio retrospectivo de Taiwán y estudios prospectivos de EE.UU. y Finlandia reportaron un aumento del riesgo. Por otro lado, otros estudios prospectivos de España y EE.UU. no lograron replicar estos resultados. Además, un estudio japonés retrospectivo mostró un efecto protector de 0.38 para la $\mathrm{DM} 2^{13}$. En general, esta evidencia sugiere que la etnia puede jugar un papel importante. El diseño del estudio también parece tener un papel importante. Un metaanálisis más reciente mostró que los estudios de cohorte proporcionaron pruebas sólidas de que la DM2 (OR: 1.29) se asociaba con un mayor riesgo de EP; por otro lado, los estudios de casos y controles mostraron una asociación inversa entre la DM2 y la EP (OR: 0.51$)^{8}$. En nuestro estudio, la DM2 fue algo más frecuente en el grupo de EP pero sin alcanzar significación estadística a pesar de ser un estudio de casos y controles. A nuestro saber y entender, este es el primer informe sobre la población latinoamericana y merece una mayor investigación.

Hay pocos estudios epidemiológicos que aborden la asociación entre la HTA y la EP con resultados que van desde la ausencia de asociación ${ }^{10}$ hasta un factor protector $^{13}$. Un metaanálisis reciente de Hou, et al. reportó una asociación de HTA preexistente con un mayor riesgo de desarrollo de EP ${ }^{12}$. Curiosamente, el subanálisis basado en características étnicas mostró que la mayor parte del efecto de riesgo fue el resultado de estudios asiáticos, mientras que una asociación baja o nula fue más común en estudios de población caucásica. Al respecto, nuestro estudio se suma al conocimiento actual al incluir la HTA como factor de riesgo independiente en una muestra latinoamericana.

Es posible que la relación entre la HTA y la EP no sea directa. El uso de propranolol se ha asociado con un mayor riesgo de $\mathrm{EP}^{21,22}$. Curiosamente, un estudio reciente informó un efecto protector de los betabloqueadores en la aparición de EP en pacientes no diabéticos, pero lo contrario en pacientes diabéticos con $\mathrm{EP}^{23}$.

En cuanto a la dislipidemia, nuestro estudio no encontró riesgo estadísticamente significativo después de ajustar por otras variables. Nuestros hallazgos están en línea con el estudio retrospectivo realizado por Yang, et al. ${ }^{10}$. Como en el caso de la HTA y los betabloqueadores, la asociación con dislipidemia podría estar más relacionada con el uso de estatinas que con los niveles de lípidos en sangre. Jeong, et al. reportaron que el uso de estatinas se asoció con un mayor riesgo de EP ${ }^{19}$. Por otro lado, un metaanálisis reciente mostró que la atorvastatina parece reducir el riesgo de $\mathrm{EP}^{24}$. Las interacciones entre las comorbilidades cardiometabólicas, los fármacos hipoglucemiantes, las estatinas y los hipoglucemiantes siguen siendo un tema difícil de dilucidar. 
Por último, la asociación del IMC con la EP tampoco fue concluyente. Los estudios longitudinales no sugieren una relación significativa entre el IMC y el desarrollo de la EP ${ }^{19,20}$. Los metaanálisis han sido contradictorios reportando tanto al sobrepeso como al bajo IMC como factor de riesgo ${ }^{15,16}$. La salvedad más notable de estos estudios es que las personas con peso normal y bajo peso se agruparon, por lo que no fue posible evaluar el bajo peso como un posible factor de riesgo independiente. Un estudio sobre este tema que utilizó una base de datos nacional coreana encontró un riesgo de 1.28 para la EP en el grupo con bajo peso, en comparación con sujetos con IMC nor$\mathrm{mal}^{23}$. Por otro lado, el mismo estudio informó un posible efecto protector para el grupo con sobrepeso, similar a nuestros hallazgos.

Nuestro estudio tiene varias limitaciones. Primero, los sujetos del grupo de EP eran mayores. No solo la edad es el factor de riesgo más importante para la $\mathrm{EP}$, sino que también puede ser cierto para todas las comorbilidades cardiometabólicas. Además, se encontró una diferencia en cuanto al sexo con menos mujeres en el grupo de EP. Si bien esto está en línea con los estudios epidemiológicos que informan una mayor prevalencia de EP en los hombres, especialmente en el grupo de 50 a 59 años ${ }^{25}$, no podemos descartar un sesgo de selección con mujeres más propensas a participar en el grupo de control. Debido a la gran muestra, el estudio de control pareado por sexo y edad habría sido difícil de lograr. No obstante, el modelo de regresión se ajustó para estas dos variables y tanto la HTA como la obesidad mantuvieron la significancia estadística. En segundo lugar, no se evaluó la relación temporal entre la aparición de comorbilidades y la aparición de la EP. A este respecto se puede argumentar que el momento del diagnóstico puede no ser realmente el momento de aparición de cualquiera de estas enfermedades evaluadas. La fase prodrómica de la EP puede durar hasta una década ${ }^{26}$, pero también la DM2, la HTA y la dislipidemia pueden permanecer sin diagnosticarse durante años, aunque estén presentes el estrés oxidativo y la inflamación crónica. Identificar el inicio real de la enfermedad, no el diagnóstico, requiere el uso de biomarcadores específicos a lo largo del tiempo. En tercer lugar, se utilizó un diagnóstico autoinformado y definiciones operativas amplias para definir la presencia o ausencia de todas las comorbilidades que están sujetas a un sesgo de recuerdo. Además, no se realizaron análisis de sangre; por tanto, no se pudo evaluar el control del riesgo cardiometabólico.

\section{Conclusiones}

Los sujetos con HTA tenían un mayor riesgo de tener EP, mientras que la obesidad proporcionó un menor riesgo de tener EP. La relación entre la enfermedad cardiometabólica, su tratamiento y la etiopatogenia de la EP parece ser extremadamente compleja dada la cantidad de datos contradictorios. Se requiere un estudio de cohorte prospectivo que evalúe los biomarcadores preclínicos tanto para la enfermedad cardiometabólica como para la EP para confirmar nuestros hallazgos.

\section{Financiamiento}

No se requirió financiamiento para este estudio.

\section{Conflicto de intereses}

Los autores declaran no tener ningún conflicto de intereses.

\section{Responsabilidades éticas}

Protección de personas y animales. Los autores declaran que los procedimientos seguidos se conformaron a las normas éticas del comité de experimentación humana responsable y de acuerdo con la Asociación Médica Mundial y la Declaración de Helsinki.

Confidencialidad de los datos. Los autores declaran que han seguido los protocolos de su centro de trabajo sobre la publicación de datos de pacientes.

Derecho a la privacidad y consentimiento informado. Los autores han obtenido el consentimiento informado de los pacientes y/o sujetos referidos en el artículo. Este documento obra en poder del autor de correspondencia.

\section{Bibliografía}

1. Rodríguez-Violante M, Velásquez-Pérez L, Cervantes-Arriaga A. Incidence rates of Parkinson's disease in Mexico: Analysis of 2014-2017 statistics. Rev Mex Neurocien. 2019;20:136-40.

2. Aviles-Olmos I, Limousin P, Lees A, Foltynie T. Parkinson's disease, insulin resistance and novel agents of neuroprotection. Brain. 2012;136:374-84.

3. Schapira AH, Jenner P. Etiology and pathogenesis of Parkinson's disease. Mov Disord. 2011;26:1049-55.

4. Tysnes OB, Storstein A. Epidemiology of Parkinson's disease. J Neural Transm (Vienna). 2017;124:901-5.

5. Ascherio A, Schwarzschild MA. The epidemiology of Parkinson's disease: risk factors and prevention. Lancet Neurol. 2016;15:1257-72.

6. Athauda D, Foltynie T. Insulin resistance and Parkinson's disease: A new target for disease modification? Prog Neurobiol. 2016;145-146:98-120.

7. Ong M, Foo H, Chander RJ, Wen MC, Au WL, Sitoh YY, et al. Influence of diabetes mellitus on longitudinal atrophy and cognition in Parkinson's disease. J Neurol Sci. 2017;377:122-6. 
8. Chohan H, Senkevich K, Patel RK, Bestwick JP, Jacobs BM, Bandres Ciga S, et al. Type 2 diabetes as a determinant of Parkinson's disease risk and progression. Mov Disord. 2021;36(6):1420-9.

9. Yue X, Li H, Yan H, Zhang P, Chang L, Li T. Risk of Parkinson disease in diabetes mellitus: An updated meta-analysis of population-based cohort studies. Medicine (Baltimore). 2016;95:e3549.

10. Yang YW, Hsieh TF, Li Cl, Liu CS, Lin WY, Chiang JH, et al. Increased risk of Parkinson disease with diabetes mellitus in a population-based study. Medicine (Baltimore). 2017;96:e5921.

11. De Pablo-Fernandez E, Goldacre R, Pakpoor J, Noyce AJ, Warner TT Association between diabetes and subsequent Parkinson disease: A record-linkage cohort study. Neurology. 2018:91:e139-e142.

12. Hou L, Li Q, Jiang L, Qiu H, Geng C, Hong JS, et al. Hypertension and diagnosis of Parkinson's disease: A meta-analysis of cohort studies. Front Neurol. 2018:9:162

13. Miyake Y, Tanaka K, Fukushima W, Sasaki S, Kiyohara C, Tsuboi Y, et al. Case-control study of risk of Parkinson's disease in relation to hypertension, hypercholesterolemia, and diabetes in Japan. J Neurol Sci. 2010;293:82-6.

14. Jeong SM, Jang W, Shin DW. Association of statin use with Parkinson's disease: Dose-response relationship. Mov Disord. 2019;34:1014-21.

15. Roos E, Grotta A, Yang F, Bellocco R, Ye W, Adami HO, et al. Body mass index, sitting time, and risk of Parkinson disease. Neurology. 2018;90:e1413-e1417

16. Palacios N, Gao X, McCullough ML, Jacobs EJ, Patel AV, Mayo T, et al. Obesity, diabetes, and risk of Parkinson's disease. Mov Disord. 2011;26:2253-9.
17. Chen J, Guan Z, Wang L, Song G, Ma B, Wang Y. Meta-analysis: overweight, obesity, and Parkinson's disease. Int J Endocrinol. 2014;2014:203930.

18. Wang $Y$, Wang $Y$, Li J, Zhang $Y$, Yin H, Han B. Body mass index and risk of Parkinson's disease: A dose-response meta-analysis of prospective studies. PLoS One 2015;10:e0131778.

19. Jeong S, Han K, Kim D, Rhee S, Jang W, Shin D. Body mass index, diabetes, and the risk of Parkinson's disease. Mov Disord. 2019;35:236-44

20. Postuma RB, Berg D, Stern M, Poewe W, Olanow CW, Oertel W, et al MDS clinical diagnostic criteria for Parkinson's disease. Mov Disord. 2015;30:1591-601.

21. Gronich N, Abernethy D, Auriel E, Lavi I, Rennert G, Saliba W. B2-adrenoceptor agonists and antagonists and risk of Parkinson's disease. Mov Disord. 2018;33:1465-71.

22. Koren G, Norton G, Radinsky K, Shalev V. Chronic use of $\beta$-blockers and the risk of Parkinson's disease. Clin Drug Investig. 2019;39:463-8.

23. de Germay S, Conte C, Rascol O, Montastruc J, Lapeyre-Mestre M. $\beta$-adrenoceptor drugs and Parkinson's disease: A nationwide nested case-control study. CNS Drugs. 2020;34:763-72

24. Yan J, Qiao L, Tian J, Liu A, Wu J, Huang J, et al. Effect of statins on Parkinson's disease: A systematic review and meta-analysis. Medicine (Baltimore). 2019;98:e14852.

25. Pringsheim T, Jette N, Frolkis A, Steeves TD. The prevalence of Parkinson's disease: a systematic review and meta-analysis. Mov Disord. 2014;29:1583-90.

26. Postuma RB, Berg D. Prodromal Parkinson's disease: The decade past, the decade to come. Mov Disord. 2019;34:665-75. 